University of Nebraska - Lincoln

DigitalCommons@University of Nebraska - Lincoln

11-29-1999

\title{
Lattice-Stiffening Transition in Copolymer Films of Vinylidene Fluoride (70\%) with Trifluoroethylene (30\%)
}

\author{
C.N. Borca \\ University of Nebraska-Lincoln \\ Shireen Adenwalla \\ University of Nebraska-Lincoln, sadenwalla1@unl.edu \\ Jaewu Choi \\ University of Nebraska-Lincoln, jchoi@ece.eng.wayne.edu \\ P.T. Sprungerr \\ Louisiana State University \\ Stephen Ducharme \\ University of Nebraska, sducharme1@unl.edu \\ See next page for additional authors
}

Follow this and additional works at: https://digitalcommons.unl.edu/physicsdowben

Part of the Physics Commons

Borca, C.N.; Adenwalla, Shireen; Choi, Jaewu; Sprungerr, P.T.; Ducharme, Stephen; Robertson, Lee; Palto, S.P.; Liu, Jianglai; Poulsen, Matt; Fridkin, V.M.; You, H.; and Dowben, Peter A., "Lattice-Stiffening Transition in Copolymer Films of Vinylidene Fluoride (70\%) with Trifluoroethylene (30\%)" (1999). Peter Dowben Publications. 40.

https://digitalcommons.unl.edu/physicsdowben/40

This Article is brought to you for free and open access by the Research Papers in Physics and Astronomy at DigitalCommons@University of Nebraska - Lincoln. It has been accepted for inclusion in Peter Dowben Publications by an authorized administrator of DigitalCommons@University of Nebraska - Lincoln. 


\section{Authors}

C.N. Borca, Shireen Adenwalla, Jaewu Choi, P.T. Sprungerr, Stephen Ducharme, Lee Robertson, S.P. Palto, Jianglai Liu, Matt Poulsen, V.M. Fridkin, H. You, and Peter A. Dowben 


\title{
Lattice-Stiffening Transition in Copolymer Films of Vinylidene Fluoride (70\%) with Trifluoroethylene (30\%)
}

\author{
C. N. Borca, ${ }^{1}$ S. Adenwalla, ${ }^{1}$ Jaewu Choi, ${ }^{1,2}$ P. T. Sprunger, ${ }^{2}$ Stephen Ducharme, ${ }^{1}$ Lee Robertson, ${ }^{3}$ S. P. Palto, ${ }^{4}$ \\ Jianglai Liu, ${ }^{1}$ Matt Poulsen, ${ }^{1}$ V. M. Fridkin, ${ }^{4}$ H. You, ${ }^{5}$ and P. A. Dowben ${ }^{1, *}$ \\ ${ }^{1}$ Department of Physics and Astronomy and the Center for Materials Research and Analysis, University of Nebraska, \\ Lincoln, Nebraska 68588-0111 \\ ${ }^{2}$ Center for Advanced Microstructure and Devices, Louisiana State University, 6980 Jefferson Highway, \\ Baton Rouge, Louisiana 70806 \\ ${ }^{3}$ High Flux Isotope Reactor (HFIR), Oak Ridge National Laboratory, Oak Ridge, Tennessee 37831 \\ ${ }^{4}$ Institute of Crystallography, The Russian Academy of Sciences, 59 Leninsky Prospekt, 117333 Moscow, Russia \\ ${ }^{5}$ Material Science Division 223, Argonne National Laboratory, 9700 S. Cass Avenue, Argonne, Illinois 60439
}

(Received 30 June 1999)

We report the discovery of a compressibility phase transition at $160 \mathrm{~K}$ in crystalline copolymer films of vinylidene fluoride $(70 \%)$ with trifluoroethylene $(30 \%)$. This phase transition is distinct from the known bulk ferroelectric-paraelectric phase transition at $353 \mathrm{~K}$ and surface ferroelectric phase transition at $295 \mathrm{~K}$. The new phase transition is characterized by an increase in the effective Debye temperature from 48 to $245 \mathrm{~K}$ along the $\langle 010\rangle$ direction as the temperature falls below $160 \mathrm{~K}$. This phase transition is evident in neutron scattering, $\mathrm{x}$-ray diffraction, angle-resolved photoemission, and in the dipole active phonon modes in electron energy-loss spectroscopy.

PACS numbers: 61.41.+e, 61.12.-q, 64.70.Kb

Phase transitions in crystalline insulators and insulating polymers are rare, excluding ferroelectric and dipole transitions (such as charge transfer and dipole reorientation). The ferroelectric polymer poly(vinylidene fluoride), PVDF, and its copolymers with trifluoroethylene (TrFE), $\left(\mathrm{CH}_{2}-\mathrm{CF}_{2}: \mathrm{CHF}_{-} \mathrm{CF}_{2}, 70 \%: 30 \%\right)$, are good insulators with high dielectric strength and low loss [1,2]. In the ferroelectric phase ( $T \leq 355 \mathrm{~K}$ for the bulk [2]; $T \leq 295 \mathrm{~K}$ for the surface [3]), the copolymer $P$ (VDF-TrFE, 70:30) is characterized, in electron spectroscopies, by a very low density of states at the Fermi level $[3,4]$ with no apparent Fermi level crossing of any sort [4]. The ferroelectric material is essentially a wide band gap $n$-type semiconductor [3]. Charge and orbital fluctuations [5-7] would appear to be very unlikely in this system with strong oriented dipoles and fully saturated nonconjugated bonds. Phase transitions in which the electronic structure is coupled to the dynamic motions of the lattice can leave a signature as a change in the lattice structure, the mean-square displacement of the atoms, the Debye temperature, a shift in the phonon modes, or the compressibility of the lattice. Such correlations between electronic structure and dynamic motions of the lattice are clearly evident in the manganese perovskites bulk transitions [8]. We find a lattice stiffening phase transition does occur, uniquely, for a wide band gap polymer system. This is evident in neutron diffraction and is not a ferroelectric transition.

The films of the $P(\mathrm{VDF}-\mathrm{TrFE}, 70: 30)$ copolymer were prepared using a horizontal Langmuir-Blodgett method $[9,10]$. X-ray diffraction measurements (XRD) were performed on a Rigaku rotating anode source with $\mathrm{Cu} K_{\alpha}$ radiation $(\lambda=1.54 \AA)$. The neutron diffraction measurements were taken with the HB1A diffractometer at the
High Flux Isotope Reactor (HFIR) at Oak Ridge National Laboratory. The neutron energy is fixed at $14.718 \mathrm{meV}$. For both x-rays and neutrons, the data were taken from the $\langle 010\rangle$ out of plane scans with the scattering vector perpendicular to the plane of the film, as indicated in the inset in Fig. 1. The measurements reported here were performed on a 5 monolayer (ML) sample for XRD and $100 \mathrm{ML}$ sample for neutron diffraction, though additional samples at different thicknesses were also studied. The angle-resolved photoemission spectroscopy (ARPES), on both 2 and 5 ML thick films, was undertaken at CAMD synchrotron facility. The $42 \mathrm{eV}$ radiation was dispersed by a plane mirror grating monochromator beam line. All the films were annealed to improve crystallinity as described previously [3,4]. The electron energy loss spectroscopy (EELS) was undertaken using an LK-2000 spectrometer. Thinner samples were essential for the electron spectroscopies to avoid excessive charging of the sample surface.

As seen in Fig. 1, the integrated intensity of the diffracted $\langle 010\rangle$ neutron peak varies substantially with temperature. As has been successfully undertaken previously [11], we have fitted the intensity attenuation of the elastic peak to $I(T)=I_{0} \exp [-2 W(T)]$, where $W(T)$ is the Debye-Waller factor. Above $200 \mathrm{~K}$ the neutron data agree well with an effective Debye temperature of $48 \pm$ $2.4 \mathrm{~K}$, while below $100 \mathrm{~K}$ the results are consistent with an effective Debye temperature of $250 \pm 12.5 \mathrm{~K}$. Above $200 \mathrm{~K}$ the effective Debye temperature $(48 \pm 2.4 \mathrm{~K})$ is in better agreement with the effective Debye temperature for $P(\mathrm{VDF}-\mathrm{TrEF})$, determined from photoemission $(53 \pm 11 \mathrm{~K})[11]$, than was the case for previous neutron scattering measurements [11] (which were influenced by 


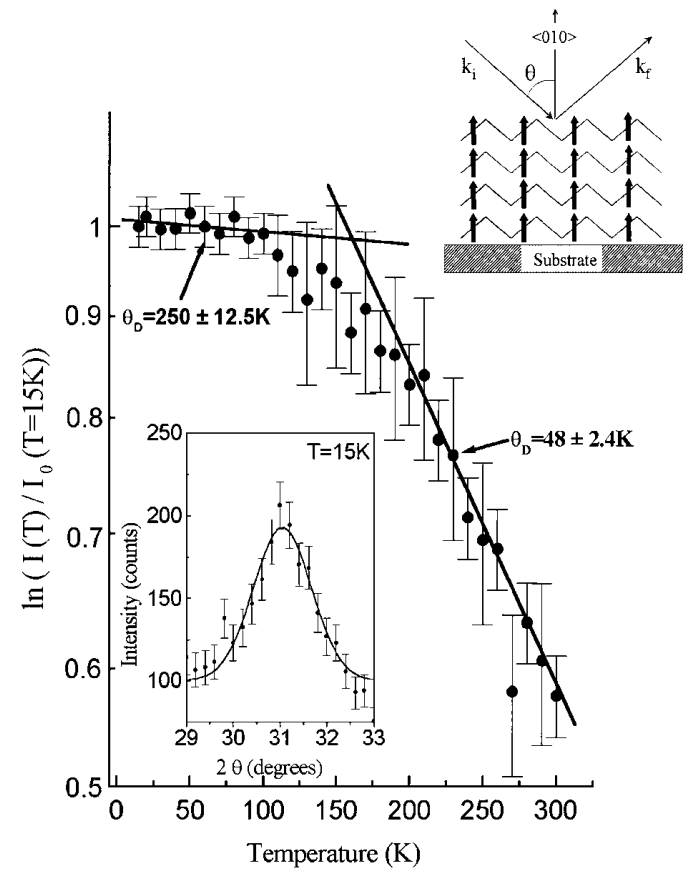

FIG. 1. Neutron scattering intensities of the interlayer $\langle 010\rangle$, $d \approx 4.5 \AA$, elastic peak plotted as a function of temperature for a $100 \mathrm{ML}$ thick film. The data are fitted to the intensity variation for an effective Debye temperature of $48 \mathrm{~K}$ above $200 \mathrm{~K}$ and $250 \mathrm{~K}$ below $100 \mathrm{~K}$, as indicated. One inset at the bottom shows the neutron diffraction peak widths and position at $15 \mathrm{~K}$. The inset at the top shows the schematic diagram of the copolymer chain structure and dipole orientation.

the phase transition at about $160 \mathrm{~K})$. From the integrated intensity of the elastic peak in neutron scattering (indicated in the insets in Fig. 1), we can estimate the change in the mean-square displacement of the lattice across this transition at about $160 \mathrm{~K}$, as seen in Fig. 2(a). This indicates a dramatic decrease in compressibility and a stiffer lattice below about $160 \mathrm{~K}$.

As shown in Figs. 2(b), the amplitude in X-ray diffraction diminishes at (or very close to) $160 \mathrm{~K}$. The width of this transition is very narrow, as can be seen by the small temperature range over which the drop in amplitude occurs. The XRD indicates that fluctuations in the electron cloud, normal to the film, are restricted to the vicinity of the transition where the change in the mean-square displacement of the lattice [Fig. 2(a)] is greatest.

The integrated intensity of the $\langle 010\rangle \mathrm{x}$-ray peak, however, does not show a clear change in slope at $160 \mathrm{~K}$ and is consistent with the high temperature bulk Debye temperature of $250 \mathrm{~K}$. This suggests that the electron cloud fluctuations normal to the film (but not necessarily inplane) are generally weak, except near the transition. The $d$ spacing (given by the position of the $\langle 010\rangle$ peak) varies linearly with temperature both in x-ray and neutron scattering as seen in Fig. 2(c), providing no indication of the transition at about $160 \mathrm{~K}$. (Differences in the $d$ spacing, with temperature, between neutron scattering and x-ray diffrac-

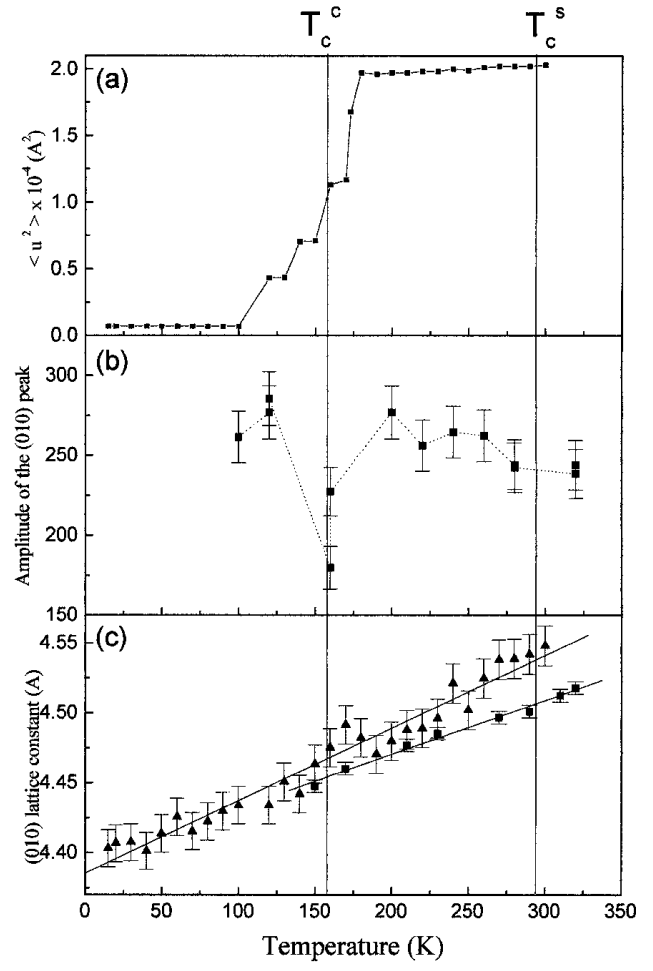

FIG. 2. (a) The mean-square displacement obtained from neutron diffraction from a 100 monolayer film. (b) The x-ray diffraction data scattering intensity is shown for a 5 monolayer film. (c) The change of the layer spacing along $\langle 010\rangle$ direction from neutron scattering $(\boldsymbol{\Delta})$ and from x-ray scattering ( $\boldsymbol{\square})$ of $P(\mathrm{VDF}-\mathrm{TrFE}), T_{\mathrm{C}}^{\mathrm{C}}=160 \mathrm{~K}$ identifies the lattice stiffening transition (the compressibility transition), while $T_{\mathrm{C}}^{\mathrm{S}}=295 \mathrm{~K}$ is the surface ferroelectric phase transition described elsewhere [2-4]. The bulk ferroelectric transition is at $353 \mathrm{~K}[2,9]$.

tion may be a consequence of the different sample film thicknesses).

The bulk ferroelectric-paraelectric phase transition in $P(\mathrm{VDF}-\mathrm{TrFE})$ has long been associated with bulk structural changes and the appearance of piezoelectric and pyroelectric effects $[12,13]$. The bulk ferroelectric $\beta$ phase, occurring below $80{ }^{\circ} \mathrm{C}$ for $P(\mathrm{VDF}-\mathrm{TrFE})$ is characterized by a structure where the polymer chains have alltrans conformation. Each monomer $\left(\mathrm{CH}_{2}-\mathrm{CF}_{2}\right)$ is aligned roughly perpendicular to the polymer chain and has its own permanent dipole moment, and normal to the surface [3,4] (inset to Fig. 1). A major conformational change of this ferroelectric $\beta$-phase structure at about $160 \mathrm{~K}$ can be precluded by the absence of a dramatic change in the $d$ spacing in the vicinity of the transition [Fig. 2(c)]. An ordered conformational change would change the $d$ spacing by $1 \%$ or more, which would be evident in Fig. 2(c). The dielectric properties normal to the films show no significant change or anomaly, consistent with these postulates.

Dynamical Jahn-Teller distortions [14] are significant in the Na doped $P(\mathrm{VDF}-\mathrm{TrFE})$ [15] and may be applicable to the undoped system as well. Fluctuations that lead to charge inhomogeneties give rise to a large compressibility 
and a soft lattice in one-dimensional models [5,6] basically the very soft lattice could have small variations of the on-site Coulomb interaction [6,7]. This can occur for an insulating system. Charge and/or orbital ordering, that occurs as the Jahn-Teller modes are frozen out [6,7], along the chains of $P$ (VDF-TrFE) could lead to an increase of the in-plane dielectric properties. This would not be apparent in the dielectric measurements and for scattering vectors normal to the film, but should be apparent in photoemission (apparent electron structure) and in the electron energy loss (the phonon modes).

We observe enhanced insulating properties in photoemission in the form of binding energy shifts of the valence bands. The shift of the valence bands in photoemission to higher binding energies is expected of final state effects [16] associated with an increasingly smaller in-plane electron screening parameter [17] or a surface photovoltage effect $[17,18]$ or an $n$-type semiconductor [such as $P$ (VDFTrFE) [3] ]. Given that all the valence band peaks shift together and that there is no change in symmetry, initial state effects seem unlikely, but cannot be excluded, as there is a small shift in the unoccupied levels at this temperature [3,4]. In Fig. 3, we show the valence band photoemission for normal emission (zero in-plane wave vector, $k_{\|}=0$ ). The dramatic increase in the photoemission binding energies begins below $210 \mathrm{~K}$. This is consistent with the onset (Fig. 1), at the high temperature end, of the lattice stiffening transition at about $200 \mathrm{~K}$. The small discrepancy in the onset in temperature between neutron scattering and photoemission data is likely due to the width of the transition

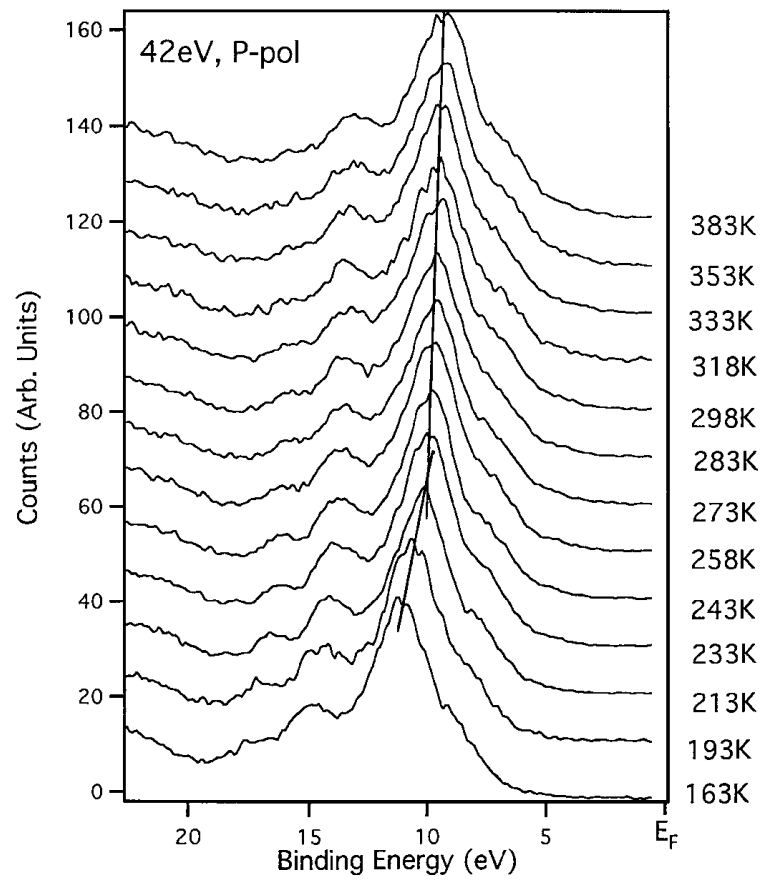

FIG. 3. The angle-resolved valence band photoemission spectra taken at normal emission as a function of temperature from $P(\mathrm{VDF}-\mathrm{TrFE})$. The photon energy is $42 \mathrm{eV}$. and different sensitivities of the two methods, especially as surface contributions cannot be excluded from photoemission. In any case, the photoemission binding energy shifts to higher binding energies, that begin to occur with decreasing temperature, are quite distinct from the known surface and bulk ferroelectric phase transitions, as summarized in Fig. 4(b).

The phase transition at about $160 \mathrm{~K}$ is also evident in EELS. EELS shows a dramatic increase, with decreasing temperature between 170 and $100 \mathrm{~K}$, in the dipole active optical vibrational modes at about 450 and $1150 \mathrm{~cm}^{-1}$ discussed in detail elsewhere [19]. This is particularly true of the mode at $450 \mathrm{~cm}^{-1}$, as plotted in Fig. 4(a). The width of these dipole active optical modes also decreases with decreasing temperature between 170 and $100 \mathrm{~K}$ as indicated in Fig. 4(a). This is consistent with a decrease in the mean square displacement of the dipoles and a lattice stiffening at about $160 \mathrm{~K}$, as is most clearly indicated by the neutron scattering data (Fig. 1).

Charge fluctuations near the $160 \mathrm{~K}$ compressibility transition are indicated by the intensity loss in XRD

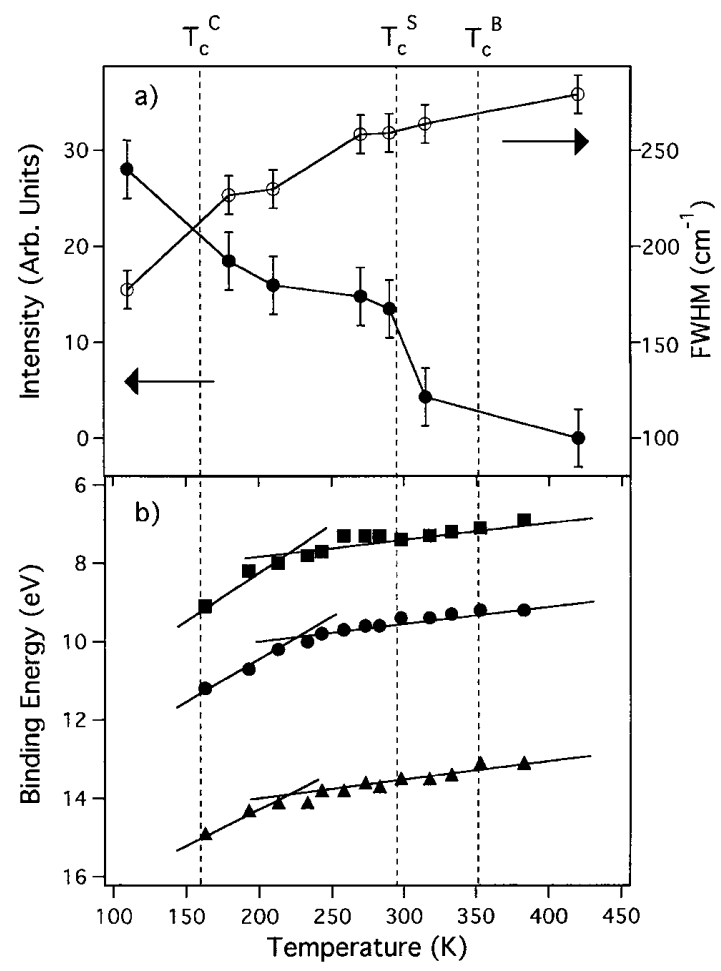

FIG. 4. The intensity of the approximately $450 \mathrm{~cm}^{-1}$ dipole active optical mode () as a function of temperature is plotted along with the width of the approximately $1150 \mathrm{~cm}^{-1}$ dipole active optical mode $(\bigcirc)$, as determined by electron energy loss, in panel (a). The data are taken in the specular scattering geometry mirror plane along the chains. The binding energy shift of the principal photoemission valence bands $(\mathbf{O}, \boldsymbol{\square}, \boldsymbol{\Lambda})$ has been plotted in panel (b). Lines are drawn to guide the eye, $T_{\mathrm{C}}^{\mathrm{C}}=160 \mathrm{~K}$ identifies the lattice stiffening transition (the compressibility transition), while $T_{\mathrm{C}}^{\mathrm{S}}=295 \mathrm{~K}$ is the surface ferroelectric phase transition described elsewhere [2-4], and the bulk ferroelectric transition $T_{\mathrm{C}}^{\mathrm{B}}=353 \mathrm{~K}[2,9]$. 
[Fig. 2(b)] that has no counterpart in the neutron diffraction intensity. Large, incoherent, charge fluctuations are certainly expected in the vicinity of a phase transition from Landau [20]. There is no reason why such fluctuations cannot occur on a length scale so small that there is little influence on the generally excellent insulating properties of this $P(\mathrm{VDF}-\mathrm{TrEF})$ copolymer material. Such phonon mediated charge fluctuations could be dramatically enhanced (or suppressed) in an excitation spectroscopy (such as photoemission) [21].

A Jahn-Teller phonon mode coupled to local charge or orbital fluctuations $[7,8]$ seems a likely driving mechanism for this transition at about $160 \mathrm{~K}$ in our crystalline films of $P(\mathrm{VDF}-\mathrm{TrFE})$. We speculate that well below the lattice stiffening transition at about $160 \mathrm{~K}$ that change and/or orbital inhomogeneities locally order and freeze much like the orbital charge ordering observed in the manganites [7,8]. Unfortunately, we do not have the data to distinguish between a change in the length scale or a change in the time scale of the postulated local chargeorbital ordering that occurs across this lattice stiffening transition in $P$ (VDF-TrFE) at about $160 \mathrm{~K}$.

The phase transition in $P(\mathrm{VDF}-\mathrm{TrFE})$ at $160 \mathrm{~K}$ is not driven by a traditional charge density wave. Coupled lattice distortions and changes in metallicity are well known for "one"-dimensional highly conjugated organic systems. Changes in the band structure and structure, with decreasing temperature, have been recently observed in TTFTCNQ (tetrathiafulvalene-tetracyanoquinodimethane) [22] and the Bechgaard salts [23]. These phase transitions are driven by strong electron-phonon coupling that affects the density of states near the Fermi energy. In fact, these Peierls-like phase transitions are driven by a decrease in the density of states near or close to the Fermi level with decreasing temperature and should be accompanied by a lattice distortion. This type of transition is not expected in a nonconjugated system like $P(\mathrm{VDF}-\mathrm{TrFE})$ with a low or nonexistent density of states near the Fermi level which exhibits a negligible change in the lattice along the $\langle 010\rangle$ direction across the transition at $160 \mathrm{~K}$.

In conclusion, we have found compelling evidence of a lattice stiffening transition at about $160 \mathrm{~K}$ in $P(\mathrm{VDF}-$ TrFE) by four different techniques: neutron diffraction, x-ray diffraction, photoemission, and electron energy loss. Well above $160 \mathrm{~K}$, we may have strong in-plane electron-phonon coupling (on a local length scale) in the absence of any appreciable density of states at or near the Fermi energy. Charge fluctuations certainly occur at the transition, as shown by $x$-ray scattering. The transition in Langmuir-Blodgett crystalline $P(\mathrm{VDF}-\mathrm{TrFE})$ at $160 \mathrm{~K}$ is novel in its behavior and distinct from most observed one-dimensional organic systems.

This work was supported by the ONR, the NSF, and AFOSR Contract No. F30602-98-C-0105 to the W. M. Keck Center for Molecular Electronics, Subcontract
No. 3531141. Palto and Fridkin were supported by the Russian Foundation for Research (No. 99-02-16484) and the Inco-Copernicus Program (No. IC15-CT96-0744). H. You is supported by the DOE under Contract No. W-31109-ENG-38. The authors acknowledge the assistance of Jens Braun and E. W. Plummer with the EELS measurements at ORNL.

*Corresponding author.

Email address: pdowben@unl.edu

[1] The Applications of Ferroelectric Polymers, edited by T. T. Wang, J.M. Herbert, and A.M. Glass (Chapman Hall, New York, 1988).

[2] A. V. Bune et al., Nature (London) 391, 874-877 (1998); S. Ducharme et al., Phys. Rev. B 57, 25 (1998).

[3] Jaewu Choi et al., Phys. Rev. Lett. 80, 1328 (1998).

[4] Jaewu Choi et al., Phys. Lett. A 249, 505 (1998).

[5] M.H. Lemée-Cailleau et al., Phys. Rev. Lett. 79, 1690 (1997).

[6] A. Moreo, S. Yunoki, and E. Dagatto, Science 283, 2034 (1999).

[7] I. S. Elfimov, V.I. Anisomov, and G. A. Sawatzky, Phys. Rev. Lett. 82, 4264 (1999).

[8] P. Dai et al., Phys. Rev. B 54, R3694 (1996); P. Dai et al., Solid State Commun. 100, 865 (1996), and references therein.

[9] S. Palto et al., Ferro. Lett. 19, 65 (1995).

[10] A. Sorokin, S. Palto, L. Blinov, V. Fridkin, and S. Yudin, Mol. Mater. 6, 61 (1996).

[11] C. N. Borca et al., Appl. Phys. Lett. 74, 347 (1999).

[12] Takeo Furukawa, Phase Transit. 18, 143 (1989).

[13] G. Thomas Davis, in The Applications of Ferroelectric Polymers, edited by T. T. Wang, J. M. Herbert, and A. M. Glass (Bell \& Bain Ltd., Glasgow, 1988), Chap. 4, p. 37.

[14] G. Santoro, L. Guidoni, A. Parola, and E. Tosatti, Phys. Rev. B 55, 16168 (1997).

[15] Jaewu Choi et al., Phys. Rev. B 59, 1819 (1999).

[16] J. E. Ortega, F. J. Himpsel, D. Li, and P. A. Dowben, Solid State Commun. 91, 807 (1994); D. N. McIlroy, J. Zhang, P. A. Dowben, and D. A. Heskett, Mater. Sci. Eng. A217/ 218, 64 (1996).

[17] Dongqi Li, Jiandi Zhang, Sunwoo Lee, and P. A. Dowben, Phys. Rev. B 45, 11876 (1992).

[18] J.E. Demuth, W. J. Thompson, N. J. DiNardo, and R. Imbihl, Phys. Rev. Lett. 56, 1408 (1986); M. Alonso, R. Cimino, and K. Horn, Phys. Rev. Lett. 64, 1947 (1990); M. H. Hecht, Phys. Rev. B 4, 7918 (1990).

[19] Jaewu Choi et al. (to be published).

[20] L.D. Landau and E. M. Lifshitz, Statistical Physics, Course of Theoretical Physics Vol. 5 (Pergamon Press, New York, 1969); A.P. Levanyuk and D. G. Sannikov, Sov. Phys. Usp. 17, 199 (1974).

[21] D. L. Lin, X. Li, Z. D. Li, and T.F. George, Phys. Lett. A 159, 369 (1991); X. Xia, X.-S. Li, D. L. Lin, and T.F. George, Phys. Rev. B 42, 4790 (1990).

[22] F. Zwick et al., Phys. Rev. Lett. 81, 2974 (1998).

[23] F. Zwick et al., Phys. Rev. Lett. 79, 3982 (1997). 